\title{
Diversité des espèces de cultures maraîchères et d'adventices hôtes de l'acarien tarsonème Polyphagotarsonemus latus Banks (Acari : Tarsonemidae) sur quelques périmètres maraîchers du Sud-Bénin
}

\author{
ADANGO Etienne ${ }^{1,3 *}$, ONZO Alexis ${ }^{2} \&$ PADONOU U. A. V. Lionel${ }^{2}$ \\ ${ }_{1}^{1}$ Direction de la Production Végétale, Ministère de l'Agriculture, de l'Elevage et de la Pêche ; République du Bénin \\ 2 Faculté d'Agronomie. Laboratoire de Phytotechnie, d'Amélioration et de Protection des Plantes (LaPAPP); \\ Université de Parakou, République du Bénin \\ ${ }^{3}$ Ecole Doctorale des Sciences Agronomiques et de l'Eau (EDSAE), Université de Parakou, République du Bénin \\ *Auteur correspondant adangoeti@yahoo.fr
}

Original submitted in on 28 th February 2020. Published online at www.m.elewa.org/journals/ on 31st May 2020

https://doi.org/10.35759/JABs.149.5

\section{RESUME}

Objectifs. La présente étude vise une protection écologiquement durable des cultures maraîchères contre l'acarien ravageur Polyphagotarsonemus latus Banks (acarien jaune, ou tarsonème trapu), à travers une meilleure connaissance de ses plantes hôtes qui, potentiellement, lui servent de réservoirs desquels il migre pour infester les cultures sur quelques périmètres maraîchers du Sud-Bénin.

Méthodologie et résultats : Quinze sites maraîchers à travers cinq communes du Sud-Bénin ont été prospectés entre Octobre et Décembre 2018 pour la présence du ravageur et d'éventuels prédateurs. Trois prospections, soit une fois par mois, ont été faites par site au cours desquelles toutes les espèces de cultures maraîchères et les principaux adventices rencontrés ont été échantillonnés. Ainsi, 5 plants ont été tirés au hasard par espèce végétale et sur chaque plant, 3 feuilles apicales ont été collectées et examinées sous loupe binoculaire pour déterminer la densité par feuille de $P$. latus et des acariens prédateurs. Des résultats de cette étude, il ressort que 15 espèces de cultures maraîchères appartenant à 8 familles botaniques et 5 espèces d'adventices appartenant à 4 familles ont hébergé $P$. latus sur l'ensemble des sites prospectés. En revanche les acariens prédateurs étaient très rares.

Conclusion et application des résultats : Cucumis melo L., Abelmoschus esculentus (L.) Moench, Cucumis sativus L., Ocimum gratissimum L., Solanum macrocarpon L., Gymnanthemum amygdalinum Delile, Spinacia oleracea L., Solanum aethiopicum L., Capsicum frutescens L, Cucurbita pepo L., Amaranthus cruentus L., Solanum lycopersicum L., Celosia argentea L., Corchorus olitorus L. et Lens culinaris Medik pour les cultures maraîchères; et Laportea aestuans L., Acalypha indica Forsk, Tridax procumbens L., Cleome viscosa L. et Lactuca taraxacifolia (Willd.) Schum pour les adventices, sont des hôtes de P. latus. Ainsi, des adventices maintiennent des populations de $P$. latus sur les périmètres maraîchers et contribuent à l'infestation des cultures dès leur installation. II importe donc aux maraîchers d'avoir une bonne connaissance des planteshôtes de $P$. latus afin d'assurer une gestion rationnelle des adventices, pour une protection durable des cultures sur les sites maraîchers.

Mots clés : Acarien tarsonème, cultures maraîchères hôtes de $P$. latus, adventices hôtes de $P$. latus, gestion durable de $P$. latus, acarien ravageur des Solanacées 
Diversity of vegetable crops and weed species hosting the broad mite, Polyphagotarsonemus latus Banks (Acari: Tarsonemidae) on some vegetable farms in Southern-Benin

\section{ABSTRACT}

Objectives: This study aimed at contributing to an ecologically sustainable protection of vegetable crops against the broad mite Polyphagotarsonemus latus Banks, through a better knowledge of its host-plants that serve as reservoir from which the mite pest migrate to infest crops on some vegetable farms in Southern-Benin.

Methodology and results: Fifteen vegetable farms distributed across five municipalities in southern Benin were surveyed from October to December 2018, for the presence of the mite pest and its possible predators. Three visits at the frequency of one per month were made per site and during which all the vegetable crops and the main weeds species encountered were sampled. Per vegetable species, 5 plants were selected at random, and on each of these plants, 3 apical leaves were collected and examined under a binocular microscope to determine the density per leaf of $P$. latus and predatory mites. Our results revealed that from all the 15 sites surveyed, 15 vegetable crop species belonging to 8 botanical families; and 5 weed species belonging to 4 families were recorded as hosting $P$. latus, whereas predatory mites were very scarce.

Conclusions and application of findings: Cucumis melo L., Abelmoschus esculentus (L.) Moench, Cucumis sativus L., Ocimum gratissimum L., Solanum macrocarpon L., Gymnanthemum amygdalinum Delile, Spinacia oleracea L., Solanum aethiopicum L., Capsicum frutescens L, Cucurbita pepo L., Amaranthus cruentus L., Solanum lycopersicum L., Celosia argentea L., Corchorus olitorus L. and Lens culinaris Medik for vegetable crops; and Laportea aestuans L., Acalypha indica Forsk, Tridax procumbens L., Cleome viscosa L. and Lactuca taraxacifolia (Willd.) Schum for weeds were host-plants of $P$. latus. Therefore, weeds can maintain $P$. latus populations on vegetable farms thereby contributing to the infestation of vegetable crop as soon as they sprout. It urges, therefore, for vegetable growers to have a good knowledge of host-plants of $P$. latus to ensure a rational management of weeds for a sustainable crop protection on their farm.

Keywords : Broad mite, vegetable crops hosting $P$. latus, weed species hosting $P$. latus, sustainable management of $P$. latus, mite pest of Solanaceous plants

\section{INTRODUCTION}

La production maraîchère, surtout celle des légumes-feuilles, occupe une place importante dans les activités agricoles développées autour des principales villes du Sud-Bénin (AssogbaKomlan et al., 2007). Elle joue un rôle primordial dans la sécurité alimentaire et nutritionnelle ainsi que dans la lutte contre la pauvreté en milieu urbain, notamment à travers sa contribution à la création d'emplois pour les jeunes et les femmes. Cependant, la production des cultures maraîchères est confrontée à de nombreuses contraintes, principalement à la pression des bioagresseurs. Parmi les ravageurs-clés recensés sur ces cultures figurent les acariens dont l'acarien tarsonème Polyphagotarsonemus latus Banks (Acari : Tarsonemidae). Cet acarien polyphage est rencontré sur des espèces végétales appartenant à une soixantaine de familles dans les régions tropicales et subtropicales à travers le monde (Gerson, 1992 ; Cross-Romero et Peña, 1998; Moraes et Flechtmann, 2008). Au Bénin, malgré le grand nombre d'espèces de cultures maraîchères produites, peu d'informations relatives à celles susceptibles d'héberger ou d'être attaquées par cet acarien polyphage sont disponibles. Cependant, il est largement reconnu que $P$. latus affectionne les cultures maraîchères de la famille des Solanaceae et particulièrement les espèces des genres Solanum et Capsicum sur lesquelles il cause de sérieux dégâts (Bordat et Goudégnon, 1991 ; Adango et al., 2006 ; 2007 ; Tossounon et Onzo, 2015). Cet acarien au corps ovale, translucide et brillant (Peña et Campbell, 2005), est quasiment invisible à l'œil nu. II se nourrit sur 
les jeunes feuilles et sur l'épiderme des fruits en formation, provoquant ainsi des distorsions irréversibles des plantes et des craquelures sur les fruits. Sa population croit d'autant plus fortement que les conditions sont relativement plus douces et plus humides (Jones et Brown, 1983); d'où sa préférence pour les périmètres maraîchers à cause des arrosages réguliers auxquels ils sont soumis. $\mathrm{Ce}$ tarsonème constitue une véritable contrainte à la production de la grande morelle Solanum macrocarpon L. (communément appelé «Gboma au Bénin »), un légume-feuilles de grande consommation au Bénin (Bordat et Goudégnon, 1991 ; Adango et al., 2006). Pour contrôler les dégâts de ce ravageur, la lutte chimique basée sur l'utilisation abusive et inappropriée des pesticides chimiques est la méthode de gestion la plus largement utilisée par les producteurs maraîchers (Sikirou et al., 2001; Assogba-Komlan et al., 2007), bien qu'il fût prouvé que cette méthode engendre des conséquences désastreuses, aussi bien sur l'environnement que sur la santé des humains (Assogba-Komlan et al., 2007 ; Mondédji et al., 2015). En effet, il a été détecté des résidus de pesticides notamment des organochlorés à des teneurs supérieures aux normes Codex dans les cultures maraîchères au Bénin (Assogba-Komlan et al., 2007), et ces résidus sont plus importants dans les feuilles de $S$. macrocarpon que dans les autres légumes-feuilles (Ahouangninou et al., 2013). Dès lors, il devient impérieux de trouver des

\section{MATERIEL ET METHODES}

Zone d'étude : L'étude a été conduite sur des sites maraîchers situés dans cinq communes du Sud-Bénin à savoir : Akpro-Missérété (lat. $6^{\circ} 33^{\prime} 45^{\prime \prime} \mathrm{N}$, long. $2^{\circ}$ $35^{\prime} 77^{\prime \prime} \mathrm{E}$ ), Porto-Novo (lat. $6^{\circ} 29^{\prime} 49.999^{\prime \prime} \mathrm{N}$, long. $2^{\circ} 36^{\prime}$ 18" E), Sèmè-Kpodji (lat. $6^{\circ} 22^{\prime} 0$ " N, long. $2^{\circ} 37^{\prime} 0^{\prime \prime}$ E) dans le Département de l'Ouémé ; Abomey-Calavi (lat. $6^{\circ} 27^{\prime} 1.089 " \mathrm{~N}$, long. $\left.2^{\circ} 20^{\prime} 48.55^{\prime \prime} \mathrm{E}\right)$ dans le Département de l'Atlantique et Grand-Popo (lat. $6^{\circ} 15^{\prime}$ $50.157^{\prime \prime} \mathrm{N}$, long. $1^{\circ} 44^{\prime} 30.171 " \mathrm{E}$ ) dans le Département du Mono. Au total, 15 sites maraîchers ont été parcourus à raison de trois sites par commune durant trois mois, d'Octobre à Décembre 2018. A AkproMissérété, les sites de Blèwan 1, Blèwan 2 et Blèwan 3 ont été prospectés ; à Porto-Novo, ce sont les sites de Houinlinda, Gbècon et Akron ; à Sèmè-Kpodji, c'est alternatives à la lutte chimique, particulièrement sur les légumes-feuilles, vu leur cycle de production très court comparativement aux autres types de cultures maraîchères. La meilleure lutte étant la prévention, les méthodes empêchant le ravageur de se retrouver sur les cultures sont d'une importance capitale. Cependant, la mise en œuvre de telles mesures passe par une bonne connaissance de l'influence de l'agencement spatial des végétaux autour de la plante-hôte, sur son infestation par le ravageur. En effet, pour des ravageurs minuscules tels que les acariens aux capacités locomotives limitées, les plantes hôtes ne constituent pas seulement des sources d'alimentation mais sont aussi un moyen pour assurer leur survie sur des générations (Alagarmalai et al., 2009), puisque les plantes du voisinage peuvent servir de réservoir au ravageur ; ce qui accélérerait son contact avec la culture cible. Ainsi donc, la bonne connaissance des plantes-hôtes de $P$. latus pourrait aider à mettre en place des mesures de lutte préventives afin d'éviter que ce ravageur cause des dégâts sur la culture-cible, en l'occurrence, S. macrocarpon ou d'autres cultures maraîchères. C'est dans cette optique que la présente étude a été initiée avec pour objectif principal l'inventaire des espèces de cultures maraîchères et d'adventices hôtes de l'acarien ravageur $P$. latus sur quelques périmètres maraîchers du Sud-Bénin.

Sika, CoJeMas et ASCOMAB qui ont été visités; à Abomey-Calavi ce sont les sites de AMACAC, FelBeLBénin et Togba-maraîchage, tandis qu'à Grand-Popo, les prospections ont porté sur les sites de Zivilatidji 1 , Zivilatidji 2 et Zivilatidji 3. Tous ces sites sont caractérisés par deux saisons des pluies (une grande et une petite), intercalées par deux saisons sèches.

Matériel d'étude : Le matériel végétal est constitué de toutes les espèces de cultures maraîchères produites et présentes sur les différents sites inspectés ainsi que des espèces d'adventices qui y sont rencontrées au cours des différentes prospections effectuées. Quant au matériel animal, il est constitué de l'acarien tarsonème $P$. latus et des acariens prédateurs de la famille Phytoseiidae rencontrés sur les sites. 
Echantillonnage et collecte des données: Trois visites ont été effectuées sur chaque site prospecté, soit une visite par mois. Sur chaque site et sur chaque espèce de cultures maraichères rencontrée, 5 plants ont été choisis au hasard, et sur chacun de ces plants, il a été collecté 3 feuilles apicales, puisque $P$. latus affectionne les jeunes feuilles. Seuls les plants situés sur les lignes centrales des parcelles ont été échantillonnés afin d'éviter les effets de bordure. Les espèces d'adventices dominantes présentes sur chaque site ont été elles-aussi échantillonnées et collectées suivant la même méthodologie que celle utilisée pour les espèces de cultures maraîchères. Des photos des adventices ont été prises pour leur identification. Lors de la collecte, les symptômes de dégâts observés sur les différentes espèces végétales ont également été photographiés. Chaque échantillon d'espèce végétale collectée sur un site donné a été conservé dans des emballages et ramené au laboratoire pour être observé sous une loupe binoculaire (marque Wild Heerbrugg de grossissement X10 pour les oculaires, et X6,4; X16 ou X40 pour les objectifs), le même jour. Les observations ont été faites feuille par feuille et par espèces végétales collectées pour y constater la présence ou non de $P$. latus et $y$ dénombrer leur effectif ainsi que celui des différentes espèces d'acarien prédateur rencontrées. Seuls les stades mobiles de $P$. latus et des prédateurs ont fait l'objet de comptage. Les spécimens de Phytoseiidae ont été prélevés et conservés dans de l'éthanol à $70 \%$ et étiquetés pour leur identification au laboratoire d'Acarologie de l'Institut International d'Agriculture Tropicale, station du Bénin (IITA-Bénin) à Cotonou.

\section{RESULTATS}

Espèces maraîchères et adventices hôtes de $P$. latus: Sur l'ensemble des 15 sites maraîchers prospectés, 21 espèces de cultures maraîchères appartenant à 13 familles botaniques et 14 espèces d'adventices issues de 11 familles botaniques ont été collectées et examinées. De cet ensemble, l'acarien
L'identification des espèces de cultures maraîchères a été faite directement sur les sites avec l'aide des maraîchers et à l'aide du manuel « Légumes Africains indigènes » de Schippers (2000). Quant aux espèces adventices, elles ont été identifiées à l'aide du « Guide des Adventices de l'Afrique de l'Ouest » (Akobundu et Agyakwa, 1989) et des photographies prises sur le terrain. II convient de faire remarquer que les dimensions des parcelles étaient variables d'une commune à une autre; et que sur tous les sites prospectés, des pesticides chimiques ont été considérablement utilisés par les producteurs maraîchers pour le contrôle des ravageurs.

Analyses Statistiques: A chaque date d'échantillonnage, les taux d'infestation des plants des différentes espèces ont été déterminés. Une analyse de variance (PROC GLM) a été effectuée sur les taux d'infestation ainsi que sur les densités de l'acarien ravageur et du prédateur phytoséiide afin de comparer les cultures entre-elles au sein d'un même site, et pour comparer les sites entre eux suivant leur degré d'infestation par $P$. latus. Des comparaisons similaires ont également été faites entre les adventices. Avant leur utilisation pour l'Anova, les taux d'infestation ont subi une transformation en $\arcsin \sqrt{ }(x)$ tandis que les valeurs des densités des acariens ont subi une transformation $\log _{10}(x+1)$ afin d'homogénéiser leur variance. En cas de différences significatives entre espèces végétales ou entre sites, les moyennes ont été séparées en utilisant le test de comparaison multiple de Student-Newman-Keuls (SNK). Toutes les analyses statistiques ont été réalisées avec le logiciel SAS (2009).

tarsonème $P$. latus a été retrouvé sur 15 espèces de cultures maraîchères issues de 8 familles botaniques, soit $71,43 \%$ des espèces maraîchères examinées (Tableau 1), et sur 5 espèces d'adventices appartenant à 4 familles botaniques, soit $35,71 \%$ des adventices examinées (Tableau 2). 
Adango et al., J. Appl. Biosci. 2020 Diversité des espèces de cultures maraîchères et d'adventices hôtes de l'acarien tarsonème Polyphagotarsonemus latus Banks (Acari : Tarsonemidae) sur périmètres maraîchers du Sud-Bénin

Tableau 1 : Présence/absence de $P$. latus sur les cultures maraîchères inspectées

\begin{tabular}{|c|c|c|}
\hline Familles botaniques & Espèces cultivées & Noms communs \\
\hline \multicolumn{3}{|c|}{ Plantes-hôtes de $P$. latus } \\
\hline \multirow{4}{*}{ Solanaceae } & Solanum aethiopicum L. & Aubergine africaine \\
\hline & Solanum macrocarpon L. & Gboma, grande morelle \\
\hline & Solanum lycopersicum L. & Tomate \\
\hline & Capsicum frutescens $\mathrm{L}$. & Piment \\
\hline \multirow[t]{3}{*}{ Cucurbitaceae } & Cucumis melo L. & Melon \\
\hline & Cucurbita pepo L. & Courgette \\
\hline & Cucumis sativus L. & Concombre \\
\hline Malvaceae & Abelmoschus esculentus (L.) Moench & Gombo \\
\hline Asteraceae & Gymnanthemum amygdalinum Delile & Vernonie \\
\hline \multirow{3}{*}{ Amaranthaceae } & Spinacia oleracea L. & Epinard \\
\hline & Amaranthus cruentus L. & Amarante \\
\hline & Celosia argentea L. & Célosie \\
\hline Lamiaceae & Ocimum gratissimum $\mathrm{L}$. & Basilic africain \\
\hline Fabaceae & Lens culinaris Medik & Lentille cultivée \\
\hline Tiliaceae & Corchorus olitorus L. & Crincrin \\
\hline \multicolumn{3}{|c|}{ Plantes non hôtes de P. latus } \\
\hline \multirow{2}{*}{ Apiaceae } & Daucus carota L. & Carotte \\
\hline & Petroselinum crispum (Mill.) Fuss & Persil commun \\
\hline Liliaceae & Allium cepa L. & Oignon \\
\hline Asteraceae & Lactuca sativa $\mathrm{L}$. & Laitue \\
\hline Solanaceae & Capsicum annum L. & Poivron \\
\hline Amaranthaceae & Betta vulgaris $\mathrm{L}$. & Betterave \\
\hline
\end{tabular}

Tableau 2 : espèces d'adventices hôtes et non hôtes inspectés sur les sites maraîchers au sud Bénin

\begin{tabular}{|l|l|l|}
\hline \multicolumn{2}{|l|}{ Familles botaniques } & \multicolumn{2}{|c|}{ Pspèces d'adventices } & Noms communs \\
\hline Urticaceae & Laportea aestuans (L.) Chew & Ortie tropicale \\
\hline Euphorbiaceae & Acalypha indica Forsk & Acalypha \\
\hline \multirow{2}{*}{ Asteraceae } & Tridax procumbens L. & Herbe à lapins \\
\cline { 2 - 3 } & Lactuca taraxacifolia (Willd.) Schum & Langue de vache \\
\hline Cleomaceae & Cleome viscosa L. (Cléome) & Moutarde sauvage \\
\hline \multicolumn{1}{|c|}{ Plantes non hôtes de P. latus } \\
\hline Commelinaceae & Aneilema beniniense (P.Beauv.) Kunth & Aneilema \\
\hline \multirow{3}{*}{ Amaranthaceae } & Amaranthus spinosus L. & Epinard piquant \\
\cline { 2 - 3 } & Pupalia lappacea (L.) Juss. & Pupalia \\
\cline { 2 - 3 } & Gomphrena celosioides Mart. & Gomphrena \\
\hline Euphorbiaceae & Euphorbia heterophylla L. & Herbe de lait \\
\hline Sterculiaceae & Waltheria indica L. & Waltheria \\
\hline Aizoaceae & Trianthema portulacastrum L. & Pourpier courant \\
\hline Convolvulaceae & Ipomoea tribola L. & Ipomée \\
\hline Poaceae & Andropogon gayanus Kunth & Andropogon \\
\hline
\end{tabular}


Abondance de $P$. latus et des acariens prédateurs phytoséiides sur les cultures maraîchères et les adventices

Sur les espèces cultivées : Au cours de la période de l'étude, la présence de $P$. latus a été enregistrée sur tous les sites maraîchers visités. Toutefois, la densité par feuille du ravageur a varié d'une espèce végétale à une autre et d'un site à un autre. La situation par commune se présente telle que décrite ci-dessous.

Commune d'Abomey-Calavi: Polyphagotarsonemus latus a été observé sur toutes les 6 espèces de cultures maraîchères inspectées. Cependant, les densités par feuille du ravageur ont différé significativement entre espèces cultivées (ddl $=5 ; F=27,70 ; P<0,0001$; Tableau 3). Ainsi, le basilic africain a été l'espèce la plus infestée par $P$. latus $(2,87 \pm 0,36$ mobiles/feuille). II est suivi du Gboma (2,07 $\pm 0,60$ mobiles/feuille) et de la vernonie $(1,59 \pm 0,22$ mobiles/feuille) qui ont des densités par feuille statistiquement identiques. Le niveau d'infestation de l'amarante $(0,86 \pm 0,18$ mobiles/feuille) a été statistiquement inférieur à ceux des trois premières espèces. Les espèces les moins infestées et sur lesquelles le ravageur a été rare sont le crincrin $(0,08 \pm 0,06$ mobiles/feuille) et le piment $(0,08$ $\pm 0,05$ mobile/feuille). Quant aux acariens prédateurs, leurs densités ont été très faibles, voire négligeables sur tous les sites maraîchers de la Commune d'Abomey-Calavi et n'ont donc pas fait l'objet d'une analyse statistique.

Commune de Grand-Popo: A Grand-Popo, la présence de $P$. latus a été observée sur la plupart des 6 espèces de cultures maraîchères échantillonnées, à l'exception de l'amarante et du crincrin (Tableau 3). L'analyse de variance a révélé une différence significative entre les densités par feuille enregistrées sur les différentes espèces cultivées ( $\mathrm{ddl}=5 ; F=$ 22,65 ; $P<0,0001$; Tableau 3). Sur le piment, bien que le ravageur y ait été enregistré, sa densité par feuille a été très faible $(0,10 \pm 0,06$ mobile/feuille), et donc pas statistiquement différente de zéro observée sur l'amarante et le crincrin (Tableau 4). Pour ce qui concerne la vernonie, le Gboma et la tomate, les densités moyennes par feuille ont été les plus élevées et statistiquement similaires avec, respectivement, 2,18 $\pm 0,38$ mobiles/ feuille ; $2,03 \pm 0,30$ mobiles/feuille et $1,63 \pm 0,51$ mobiles/feuille (Tableau 3). Les acariens prédateurs ont été absents sur toutes les espèces observées sauf sur la vernonie où une densité moyenne de 0,06 $\pm 0,03$ mobile/feuille a été enregistrée.

Commune d'Akpro-Missérété : Sur les périmètres maraîchers prospectés dans la commune de AkproMissérété, $P$. latus a été observé sur 4 des 6 espèces échantillonnées (Tableau 3). L'analyse de variance a révélé une différence significative de densités moyennes par feuille entre les espèces collectées (ddl $=5 ; F=23,92 ; P<0,0001 ;$ Tableau 3). La plus forte densité moyenne par feuille a été enregistrée sur le basilic $(1,79 \pm 0,24$ mobiles/feuille) suivi de la vernonie (1,41 $\pm 0,25$ mobiles/feuille) et du Gboma (1,36 $\pm 0,18$ mobiles/feuille); puis de l'amarante $(0,99 \pm 0,19$ mobile/feuille). Les acariens prédateurs ont été rares sur tous les sites.

Tableau 3 : Densités de $P$. latus par feuille d'espèce maraichères échantillonnées à Abomey-Calavi, Grand-Popo et Akpro-Missérété

\begin{tabular}{lllllll}
\hline \multicolumn{2}{l}{ Communes d'investigation } \\
\hline & \multicolumn{2}{l}{ Abomey-Calavi } & \multicolumn{2}{l}{ Grand-Popo } & \multicolumn{2}{l}{ Akpro-Missérété } \\
\hline $\begin{array}{l}\text { Espèces } \\
\text { cultivées }\end{array}$ & $\mathbf{N}$ & Moy \pm E.S & N & Moy \pm E.S & N & Moy \pm E. S \\
\hline Amarante & 135 & $0,86 \pm 0,18 \mathrm{c}$ & 75 & $0,00 \pm 0,00 \mathrm{~b}$ & 135 & $0,99 \pm 0,19 \mathrm{~b}$ \\
Basilic africain & 135 & $2,87 \pm 0,36 \mathrm{a}$ & - & - & 135 & $1,79 \pm 0,24 \mathrm{a}$ \\
Crincrin & 135 & $0,08 \pm 0,06 \mathrm{~d}$ & 15 & $0,00 \pm 0,00 \mathrm{~b}$ & 135 & $0,00 \pm 0,00 \mathrm{c}$ \\
Gboma & 135 & $2,07 \pm 0,60 \mathrm{~b}$ & 135 & $2,03 \pm 0,30 \mathrm{a}$ & 135 & $1,36 \pm 0,18 \mathrm{ab}$ \\
Piment & 90 & $0,08 \pm 0,05 \mathrm{~d}$ & 135 & $0,10 \pm 0,06 \mathrm{~b}$ & - & - \\
Vernonia & 135 & $1,59 \pm 0,22 \mathrm{~b}$ & 90 & $2,18 \pm 0,38 \mathrm{a}$ & 90 & $1,41 \pm 0,25 \mathrm{ab}$ \\
Tomate & - & - & 30 & $1,63 \pm 0,51 \mathrm{a}$ & - & - \\
Célosie & - & - & - & - & 60 & $0,00 \pm 0,00 \mathrm{c}$ \\
\hline
\end{tabular}

Dans une même colonne, les moyennes suivies d'une même lettre alphabétique ne sont pas statistiquement différentes au seuil de $5 \%$ (SNK Test). 
Commune de Porto-Novo: Dans la Commune de Porto-Novo, toutes les 13 espèces de cultures maraîchères examinées, à l'exception du piment ont été infestées par $P$. latus, avec une différence significative entre les densités moyennes enregistrées (ddl = $12 ; F=35,49 ; P<0,0001 ;$ Tableau 4). Ainsi, le melon et le gombo ont été les espèces statistiquement les plus infestées avec, respectivement, des densités moyennes de $34,13 \pm 6,23$ mobiles/feuille et de 27,87 $\pm 4,51$ mobiles/feuille. Viennent ensuite, le concombre $(10,80 \pm 3,65$ mobiles/feuille), le basilic $(4,37 \pm 0,41$ mobiles/feuille), l'aubergine $(4,16 \pm 0,84$ mobiles/feuille) dont les densités ont été statistiquement similaires. Elles sont suivies du Gboma $(2,47 \pm 0,33$ mobiles/feuille) et de la vernonie $(2,44 \pm$ 0,40 mobiles/feuille) qui ont été plus modérément infestées avec des densités statistiquement similaires (Tableau 4). La célosie (1,93 $\pm 0,38$ mobiles/feuille), le crincrin $(1,18 \pm 0,22$ mobiles/feuille), la lentille $(1,13 \pm$ 0,52 mobiles/feuille), l'amarante $(0,90 \pm 0,19$ mobile/feuille) et la tomate $(0,87 \pm 0,25$ mobiles/feuille) ont été les espèces les moins infestées (Tableau 4). Les acariens prédateurs ont été rencontrés sur 7 des
13 espèces botaniques, soit $53,85 \%$ des espèces cultivées échantillonnées mais leurs densités moyennes n'ont pas été différentes de zéro.

Commune de Sèmè-Kpodji: A Sèmè-Kpodji, la présence de $P$. latus a été observée sur toutes les 10 espèces cultivées à l'exception du poivron (Tableau 4). Ici aussi, l'analyse de variance révèle que les densités moyennes enregistrées ont varié significativement entre les différentes cultures (ddl $=9 ; F=51,29 ; P<$ 0,0001 ). Ainsi, l'épinard a été l'espèce la plus infestée avec une densité moyenne de 8,27 $\pm 1,13$ mobiles/feuille. Elle est suivie du basilic $(4,87 \pm 0,63$ mobiles/feuille), du concombre (4,34 $\pm 0,48$ mobiles/feuille), du Gboma (3,85 $\pm 0,36$ mobiles/feuille), de la courgette (3,47 $\pm 0,98$ mobiles/feuille) et puis du piment (3,36 $\pm 0,35$ mobiles/feuille) sur lesquelles les densités enregistrées ont été modérées et statistiquement similaires. Les espèces les moins infestées sont: la vernonie $(1,37 \pm$ 0,22 mobiles/feuille), la tomate $(0,36 \pm 0,10$ mobiles/feuille) et l'amarante $(0,32 \pm 0,11$ mobiles/feuille) (Tableau 4). Les acariens prédateurs y ont été très rares.

Tableau 4 : Densités de $P$. latus par feuille d'espèces maraîchères échantillonnées à Porto-Novo et à Sèmè-Kpodji

\begin{tabular}{|c|c|c|c|c|}
\hline \multicolumn{5}{|c|}{ Communes d'investigation } \\
\hline & \multicolumn{2}{|c|}{ Porto-Novo } & \multicolumn{2}{|c|}{ Sèmè-Kpodji } \\
\hline Espèces cultivées & $\mathbf{N}$ & Moy \pm E. S & $\mathbf{N}$ & Moy $\pm E . S$ \\
\hline Amarante & 135 & $0,90 \pm 0,19 \mathrm{ef}$ & 135 & $0,32 \pm 0,11 d$ \\
\hline Aubergine & 45 & $4,16 \pm 0,84 c d$ & - & - \\
\hline Basilic & 135 & $4,37 \pm 0,41 b c$ & 90 & $4,87 \pm 0,63 b$ \\
\hline Celosia & 60 & $1,93 \pm 0,38 \mathrm{de}$ & - & - \\
\hline Concombre & 30 & $10,80 \pm 3,65 b$ & 134 & $4,34 \pm 0,48 b$ \\
\hline Crincrin & 135 & $1,18 \pm 0,22 e f$ & - & - \\
\hline Gboma & 135 & $2,47 \pm 0,33 \mathrm{cde}$ & 135 & $3,85 \pm 0,36 b$ \\
\hline Gombo & 15 & $27,87 \pm 4,51 \mathrm{a}$ & - & - \\
\hline Lentille & 15 & $1,13 \pm 0,52$ ef & - & - \\
\hline Melon & 15 & $34,13 \pm 6,23 a$ & - & - \\
\hline Piment & 45 & $0,00 \pm 0,00 f$ & 90 & $3,36 \pm 0,35 b$ \\
\hline Tomate & 45 & $0,87 \pm 0,25 \mathrm{ef}$ & 120 & $0,36 \pm 0,10 d$ \\
\hline Vernonia & 135 & $2,44 \pm 0,40 \mathrm{de}$ & 135 & $1,37 \pm 0,22 \mathrm{c}$ \\
\hline Courgette & - & - & 15 & $3,47 \pm 0,98 b$ \\
\hline Epinard & - & - & 30 & $8,27 \pm 1,13 a$ \\
\hline Poivron & - & - & 120 & $0,00 \pm 0,00 d$ \\
\hline
\end{tabular}

Dans une même colonne, les moyennes suivies d'une même lettre alphabétique ne sont pas statistiquement différentes au seuil de $5 \%$ (SNK Test).

Abondance de $P$. latus et des prédateurs
phytoséiides sur les adventices
Commune d'Abomey-Calavi : Toutes les 3 espèces
d'adventices collectées à Abomey-Calavi ont abrité le ravageur avec, cependant, une différence significative entre les densités par espèce ( $\mathrm{ddl}=2 ; F=20,08 ; P<$ $0,0001)$. Ainsi, la densité moyenne la plus élevée de $P$. latus a été enregistrée sur l'espèce $L$. aestuans avec 
$1,26 \pm 0,20$ mobiles/feuille, alors que celles enregistrées sur $A$. indica et $L$. taraxacifolia, ont été statistiquement similaires avec, respectivement, $0,32 \pm$ 0,11 mobile/feuille et $0,22 \pm 0,10$ mobile/feuille. Pour ce qui concerne les prédateurs, ils ont été pratiquement absents sur toutes les trois espèces observées à l'exception de $L$. aestuans sur laquelle la densité moyenne a été de $0,02 \pm 0,02$ mobile/feuille.

Commune de Grand-Popo: A Grand-Popo seules 2 des 4 espèces d'adventice collectées ont été infestées par $P$. latus. II s'agit de $L$. aestuans $(0,79 \pm 0,17$ mobile/feuille) et de $A$. indica $(0,68 \pm 0,16$ mobile/feuille). Le ravageur n'a pas été retrouvé sur Cleome viscosa et Tridax procumbens. L'analyse de variance a révélé une différence significative entre les 4 espèces (ddl $=3 ; F=12,10 ; P<0,0001$ ) avec $L$. aestuans et $A$. indica ayant des densités statistiquement similaires. Les acariens prédateurs ont été absents sur toutes les adventices collectées à Grand-Popo.

Commune d'Akpro-Missérété : A Akpro-Missérété, $P$. latus n'a été présent que sur les adventices $L$. aestuans $(0,48 \pm 0,11$ mobile/feuille) et $A$. indica $(0,30 \pm 0,10$ mobile/feuille) tandis qu'il a été absent sur Gomphrena celosioides et $T$. procumbens. L'analyse de variance a révélé une différence significative ( $\mathrm{ddl}=3 ; F=13,89$; $P<0,0001$ ) avec $L$. aestuans et $A$. indica ayant des densités statistiquement similaires. Les acariens prédateurs ont été observés uniquement sur $L$. aestuans avec une densité de 0,07 $\pm 0,03$ mobile/feuille.

\section{DISCUSSION}

Diversité des espèces végétales hôtes de $P$. latus : La bonne connaissance de l'écosystème des ravageurs et de l'ampleur des dégâts qu'ils causent aux cultures est la première étape dans la mise en place de toute stratégie de lutte durable (Lavabre, 1992 ; Ochoa et al., 1994). Ainsi, la présente étude, axée sur l'inventaire des différentes espèces de cultures maraîchères et d'adventices susceptibles d'être hôtes du tarsonème $P$. latus sur les sites maraîchers du Sud-Bénin confirme que beaucoup d'espèces maraîchères et d'adventices sont hôtes de ce ravageur (Gerson, 1992). En effet, les résultats de cette étude ont révélé qu'au moins 15 espèces de cultures maraîchères appartenant à 8 familles botaniques sont des hôtes du ravageur. Parmi ces familles, sept à savoir : Tiliaceae, Asteraceae, Amaranthaceae, Cucurbitaceae, Fabaceae, Malvaceae et Solanaceae ont déjà été rapportées par plusieurs auteurs comme familles abritant des plantes-hôtes de
Commune de Porto-Novo: A Porto-Novo une différence significative a été observée entre les 5 espèces d'adventices pour les densités par feuille de $P$. latus (ddl $=4 ; F=33,95 ; P<0,0001$ ). Ici aussi, c'est l'espèce $L$. aestuans (1,50 $\pm 0,18$ mobile/feuille) qui a connu le niveau le plus élevé d'infestation de $P$. latus devant $A$. indica $(0,29 \pm 0,09$ mobile/feuille $)$ et $T$. procumbens $(0,09 \pm 0,05$ mobile/feuille $)$. Sur les espèces $C$. viscosa et $G$. celosioides, aucune présence du ravageur n'a été enregistrée. Pour ce qui est des prédateurs, ils ont été enregistrés uniquement sur $L$. aestuans ( $\mathrm{ddl}=4 ; F=8,51 ; P<0,0001$ ), avec une densité moyenne de $0,16 \pm 0,05$ mobile/feuille, statistiquement différente de celles (nulles) des autres espèces.

Commune de Sèmè-Kpodji: A Sèmè-Kpodji, la présence de $P$. latus a été enregistrée sur les deux espèces d'adventices hôtes qui y ont été collectées à savoir $C$. viscosa et $L$. aestuans, avec des densités moyennes respectives de 0,93 $\pm 0,17$ mobile/feuille et $0,73 \pm 0,16$ mobile/feuille. Aucune différence significative n'a été révélée entre ces deux espèces végétales ( $\mathrm{ddl}=1 ; F=0,81 ; P=0,3703)$. Les acariens prédateurs ont été enregistrés uniquement sur $L$. aestuans avec une densité de 0,01 \pm 0,01 mobile/feuille. Sur l'ensemble de l'étude, les acariens prédateurs collectés appartiennent à la famille Phytoseiidae et majoritairement à l'espèce Amblyseius rykei (Pritchard \& Baker), à laquelle s'ajoutent quelques spécimens de Neoseiulus barkeri Hughes et quelques spécimens non identifiés.

P. latus (Ewing, 1939; Goff, 1987; Bordat et Goudégnon 1991; Gerson, 1992; Adango et al., 2006). En revanche, la famille Lamiaceae n'avait été rapportée par aucun de ces auteurs comme renfermant des espèces hôtes de $P$. latus. II ressort également de l'étude que dans le groupe des plantes cultivées, la famille Solanaceae est celle qui renferme le plus grand nombre d'hôtes de $P$. latus avec 4 espèces, suivie de la famille Cucurbitaceae avec 3 espèces. Ces résultats confirment ceux de Adango et al. (2006) qui ont rapporté $P$. latus sur $S$. macrocarpom et $S$. aethiopicum mais pas sur l'amarante au Sud-Bénin. Cependant, sur le poivron, reconnu comme l'une des espèces de Solanaceae les plus infestées par ce ravageur (de Coss-Romero et Peña, 1998 ; Ashraf et al., 2011), aucun individu de $P$. latus n'a été observé au cours de l'étude; ce qui est tout de même curieux et nécessite donc une explication. Les investigations faites sur le 
terrain permettent d'affirmer que l'absence de l'acarien tarsonème sur ce légume s'expliquerait par le fait que les producteurs, bien informés de sa forte sensibilité aux attaques de $P$. latus, utilisent alors abondamment des pesticides pour assurer la protection du poivron contre le ravageur. Pour ce qui concerne les adventices, les résultats de cette étude révèlent que 5 espèces appartenant à 4 familles botaniques (Urticaceae, Euphorbiaceae, Asteraceae et Cleomaceae), abritent $P$. latus. Ceci confirme les résultats de Schwartz (1977), et de Waterhouse et Norris (1987), qui ont mis en évidence l'importance des mauvaises herbes en tant que réservoirs de $P$. latus. Ainsi, les adventices sont susceptibles de constituer de potentiels foyers temporaires (Lavoipierre, 1940), desquels le ravageur peut migrer sur les cultures maraichères lorsque les conditions lui sont favorables. Sur les 4 familles d'adventices identifiées, Euphorbiaceae et Asteraceae ont déjà été signalées comme abritant des espèces hôtes de ce tarsonème (Lavoipierre, 1940 ; Gerson, 1992). En revanche, aucune espèce des familles Urticaceae et Cleomaceae n'avait jamais été rapportée (à notre connaissance), comme hôte de $P$. latus. Ces résultats confirment ainsi ceux de Gerson (1992) qui spéculait sur l'existence probable d'autres familles botaniques hôtes, non encore identifiées, de $P$. latus. Au total, sur les 35 espèces botaniques inspectées au cours de la présente étude, 21 espèces dont 6 adventices ont été identifiées comme hôte de $P$. latus, confirmant de ce fait les résultats de plusieurs études selon lesquelles ce ravageur est capable de se maintenir sur un grand nombre d'espèces végétales (Gerson, 1992 ; Peña et Campbell, 2005 ; Renkema et al., 2017) ; ce qui lui vaut le nom commun anglais de "Broad Mite ». En dehors des 4 espèces de solanacées reconnues comme hôte de $P$. latus, les autres espèces hôtes identifiées ne sont pas connues des producteurs comme étant, ou pouvant être infestées par le tarsonème. En effet, bien qu'infestées, ces espèces ne présentent généralement aucun des symptômes habituels des attaques de $P$. latus ; ce qui empêche les maraîchers de les identifier. Ces résultats confirment les observations de plusieurs auteurs selon lesquelles les symptômes des dégâts de ce ravageur peuvent varier d'une espèce à une autre à cause de la variation des réactions des plantes hôtes à ses attaques (Aubert et al., 1981 ; Gerson, 1992). Ainsi, alors que les symptômes sont bien visibles sur $A$. indica et $L$. aestuans, ils sont, par contre, difficilement perceptibles surles autres adventices hôtes du ravageur.
Abondance de $P$. latus et des prédateurs sur les espèces cultivées et les adventices: II ressort de l'examen des résultats de cette étude que les densités de population du ravageur varient aussi bien au sein des espèces maraîchères cultivées qu'au sein des adventices. Dans le groupe des espèces cultivées, le melon, le gombo, le concombre, le basilic, la grande morelle (Gboma), la vernonie et l'épinard ont été les plus infestées par $P$. latus, tandis que l'aubergine, le piment, la courgette, l'amarante, la tomate, la célosie, le crincrin et la lentille ont été les espèces les moins infestées. Quant aux adventices, l'étude a montré que ce sont $L$. aestuans et $A$. indica qui ont abrité la plus grande population de $P$. latus durant la période de l'étude. En effet, ces deux plantes sont disséminées un peu partout, poussant facilement dans et aux abords des parcelles de cultures. Dotées d'une croissance rapide du fait qu'elles ne sont pas exigeantes, elles sont généralement négligées par les producteurs qui n'effectuent pas rigoureusement les travaux d'entretien. Ainsi, ces espèces constituent des foyers temporaires pour le ravageur avant l'installation des cultures principales. Elles jouent, par conséquent, un rôle non négligeable dans la distribution de $P$. latus. La variation de la densité par feuille du ravageur d'une espèce à une autre sur un même site confirme les résultats de Alagarmalai et al. (2009) selon lesquels $P$. latus, bien que limité dans ses déplacements, est capable de choisir ses hôtes; les femelles étant beaucoup plus responsables de cette sélection. Plusieurs facteurs seraient à l'origine de ce comportement observé chez les arthropodes phytophages. II s'agit entre autres des métabolites primaires et secondaires, de même que de la morphologie et de la phénologie des plantes (Schoonhoven et al., 1998). Le fait que S. macrocarpon eût été infesté sur l'ensemble des sites prospectés contrairement à certaines espèces maraîchères cultivées laisse croire que $P$. latus a véritablement une préférence pour cette espèce qui fait partie de ses hôtes de prédilection au Bénin (Bordat et Goudégnon, 1991). S'agissant des prédateurs, l'étude a montré qu'ils ont été très rares sur les cultures maraîchères au niveau de tous les sites et viennent en soutien aux résultats rapportés par Adango et al. (2007) et Azandémè-Hounmalon et al. (2015). Ces auteurs expliquent ce constat par la forte utilisation de pesticides par les producteurs sur tous les sites maraîchers pour contrôler $P$. latus et d'autres ravageurs et/ou maladies des cultures maraîchères. En effet, les ennemis naturels des acariens, surtout les acariens prédateurs phytoséiides, succombent rapidement dans 
les écosystèmes assujettis à une forte utilisation de pesticides pour le contrôle phytosanitaire (Meyer, 1996 ; Reckhaus, 1997 ; Adango et al., 2007), vu leur plus grande sensibilité aux pesticides chimiques en comparaison au ravageur (Appert et Deuse, 1982 ; Gerson, 1992 ; McKinlay et al., 1992). Aussi, une étude réalisée par Sikirou et al. (2001) a montré que sur tous les périmètres maraîchers du Bénin, la lutte à base de produits chimiques, notamment ceux à large spectre d'action comme les pyréthrinoïdes, est la plus communément utilisée, alors que ceux-ci ne favorisent pas le maintien des prédateurs dans les écosystèmes (Brown et Shanks, 1976 ; Hassan, 1982; Wilson, 1997; Wilson et Sadras, 1997). La population d'acariens prédateurs phytoséiides rencontrés est dominée par l'espèce $A$. rykei qui, selon Zannou et Hanna (2011), n'est que le synonyme de Amblyseius swirskii (Athias-Henriot), une espèce qui, dans de récentes études au laboratoire, a démontré un fort potentiel de prédation sur l'acarien tarsonème $P$. latus (Onzo et al., 2012). La présence de $A$. rykei sur certaines plantes n'est donc pas le fait d'un hasard et

\section{REMERCIEMENTS}

Les auteurs témoignent leur gratitude aux maraîchers des différentes communes prospectées pour leur franche collaboration. Notre gratitude va à l'endroit de Mrs Wilson Daoudou et Wenceslas Kassa ainsi qu'aux techniciens du Laboratoire d'Entomologie de la Direction de la Production Végétale à Porto-Novo pour pourrait bien être un indicateur de la possibilité d'envisager la lutte biologique contre le ravageur. II se dégage des résultats de la présente étude que les adventices constituent d'importants réservoirs qui maintiennent les populations de $P$. latus sur les périmètres maraîchers en l'absence des plantes hôtes cultivées, notamment Gboma, facilitant ainsi leur colonisation par le ravageur dès qu'elles sont installées. La présence de ces adventices sur ou aux alentours des sites maraîchers doit donc être un indicateur d'une potentielle colonisation des cultures maraichères par $P$. latus. Dès lors, ce paramètre mérite d'être pris en considération dans le développement des stratégies de gestion durable de cet acarien dans le maraîchage en général et en particulier sur les solanacées comme la grande morelle S. macrocarpon (Gboma). Aussi, les producteurs ont-ils besoin d'avoir une bonne connaissance des plantes cultivées ou adventices, hôtes de ce tarsonème lors de l'établissement de leur plan de campagne afin d'assurer une meilleure protection de leurs cultures contre ce ravageur.

leur assistance dans la collecte et le dépouillement des échantillons. Sincères remerciements aux techniciens du Laboratoire d'Acarologie de l'Institut International d'Agriculture Tropicale, station du Bénin (IITA-Bénin) pour leur précieuse assistance.

\section{DECLARATION DE CONFLIT D'INTERETS}

Tous les auteurs déclarent l'existence d'aucun conflit d'intérêts.

\section{REFERENCES}

Adango, E., Onzo, A., Hanna, R., Atachi, P. \& James, B. 2006. Inventaire de la faune des acariens sur Amaranthus cruentus L. (Amaranthaceae), Solanum macrocarpom $L$. et Solanum aethiopicum L. (Solanaceae) dans le sud Bénin. International Journal of Tropical Insect Science, 26(3) : 155-165.

Adango, E., Onzo, A., Hanna, R., Atachi, P. \& James, B. 2007. Mite pests of major importance on indigenous leafy vegetables in Benin: the search for appropriate control strategies. In: M. L. Chadha, G. Kuo, C. L. L. Gowda (editors) Proceedings of the 1st International Conference on Indigenous Vegetables and Legumes. Prospectus for Fighting Poverty, Hunger and Malnutrition. Acta Horticulturae,
752, 311-318. DOI : https://doi.org/10.17660/ActaHortic.2007.752. 53.

Ahouangninou C., Fayomi E.B. \& Martin T. 2011. Évaluation des risques sanitaires et environnementaux des pratiques phytosanitaires des producteurs maraichers dans la commune rurale de Tori-Bossito (SudBénin). Cahiers Agricultures, 20(3), 216-222

Ahouangninou C. et al. 2013. Using Aedes aegypti larvae to assess pesticide contamination of soil, groundwater and vegetables. British biotechnology journal, 3(2), 143-157.

Akobundu O. I. \& Agyakwa C. W. 1989. Guide des adventices d'Afrique de l'Ouest. IITA, 522p 
Alagarmalai J., Grinberg M., Perl-Treves R. \& Soroker V. 2009. Host Selection by the Herbivorous Mite Polyphagotarsonemus latus (Acari: Tarsonemidae). Journal of Insect Behavior (2009) 22 : 375-387

Appert J. \& Deuse J. 1982. Les ravageurs des cultures vivrières et maraîchères sous les tropiques. eds Maisonneuve et Larose, Paris, France. 420p.

Ashraf A. M., Ahmed M. T., Hanafy A. R. \& Gamal M. H. 2011. Biology and control of the broad mite Polyphagotarsonemus latus (Banks, 1904) (Acari: Tarsonemidae). International Journal of Environmental Science and Engineering, 1: 26 -34 .

Assogba-Komlan F., Anihouvi P., Achigan E., Sikirou R., Boko A., Adjé C., Vodouhe, R. S. \& Ahlè V. 2007. Pratiques culturales et teneur en éléments anti-nutritionnels (nitrates et pesticides) du Solanum macrocarpum sud du Bénin. African Journal of Food Agriculture Nutrition and Development, 7(4): 1-21.

Aubert B., Lassois P. \& Marchal J. 1981. Mise en évidence des dégâts causés par Polyphagotarsonemus latus (Banks) sur papayer à l'île de la Réunion. Fruits $36: 9-24$.

Bordat, D. \& Goudegnon, E. 1991. Catalogue des principaux ravageurs des cultures maraîchères au Bénin. CIRAD (ed), Montpellier, France. $15 \mathrm{p}$.

Brown G. C. \& Shanks C. H., 1976. Mortality of two spotted mite predators caused by the systemic insecticide, carbofuran. Environmental Entomology, 5: 1155-1159.

Azandémè-Hounmalon G.Y., Affognon H.D., AssogbaKomlan F., Tamó M., Fiaboe K.K.M., Kreiter S. \& Martin T. 2015. Farmers' control practices against the invasive red spider mite, Tetranychus evansi Baker \& Pritchard in Benin. Crop Protection, 76: 53-58.

Coss-Romero M. \& Peña J.E. 1998: Relationship of broad mite (Acari: Tarsonemidae) to host phenology and injury levels in Capsicum annuum. Florida Entomologist 81: 515-526.

Ewing, H.E., 1939. A revision of the mites of the subfamily Tarsoneminae of North America, the West Indies and the Hawaiian Islands. USDA Technical Bulletins. 653, 63pp

Gerson, U. (1992). Biology and control of the broad mite. Polyphagotarsonemus latus (Banks)
(Acari: Tarsonemidae). Experimental and Applied Acarology, 13:163-178.

Goff, M.L. 1987. A catalog of the Acari of the Hawaiian Islands. University of Hawaii, Residence extension service. No. $075,75 \mathrm{p}$.

Hassan S. A. 1982. Relative tolerance of 3 different strains of predatory mite Phytoseiulus persimilis to 11 pesticides used on glasshouse crops. Zeitschrift für Angewandte Entomologie $93: 55-63$.

Lavabre E. M., 1992. Ravageurs des cultures tropicales. Maisonneuve \& Larose (eds), Paris, France, 178p.

Jones V. P. \& Brown R. D. 1983. Reproductive responses of broad mite Polyphagotarsonemus latus (Acari: Tarsonemidae) to constant temperaturehumidity regimes. Annal of the Entomological Society of America 76: 466-469.

Lavoipierre, M. M. J. 1940. Hemitarsonemus latus (Banks) (Acarina), a mite of economic importance new to South Africa. Journal of the Entomological Society of Southern Africa, 3: 116-123.

McKinlay R. G., Spaull A. M. \& Straub R. W. 1992. Pest of Solanaceous crops. In: Vegetable crops pests, McKinlay G. R. (ed) CRC Press Inc. Boston, USA, pp 263-323.

Meyer S. K. M. 1996. Mite pests and their predators on cultivated plants in southern Africa: Vegetables and Berries. ed. Isteg Scientific publication, Johannesburg, South Africa, 90p.

Moraes G.J. \& Flechtmann C.H.W. 2008. Manual de Acarologia - Acarologia básica e ácaros de plantas cultivadas no Brasil. Ribeirão Preto: Ed. Holos. 308p.

Ochoa R., Aguilar H. \& Vargas C. 1994. Phytophagous mites of Central America: an illustrated guide. Serie Técnica. Manual Técnico/ CATIE $n^{\circ} 6$. Ed. Eng., Turrialba, Costa Rica 234p.

Onzo A., Houedokoho A.F. \& Hanna, R. 2012. Potential of the predatory mite, Amblyseius swirskii to suppress the broad mite, Polyphagotarsonemus latus on the gboma eggplant, Solanum macrocarpon. Journal of Insect Science, $12, \quad 7$. https://doi.org/10.1673/031.012.0701

Peña J.E. \& Campbell C.W. 2005. Broad Mite. University of Florida, IFAS Extension http://edis.ifas.ufl.edu. $4 p$. 
Reckhaus P. 1997. Maladie et ravageurs des cultures maraîchères: à l'exemple de Madagascar, Margraf (ed.) Weikersheim, Allemagne, 402p.

Renkema J. M., LeFors J. A. et Johnson D. T. 2017. First report of broad mite (Acari: Tarsonemidae) on commercial Strawberry in Florida. Florida Entomologist, 100(4) : 804806.

Schippers R. R. 2000. Légumes africains indigènes : Présentation des espèces cultivées. Margraf \& CTA (eds), Weikersheim, Allemagne, 482p.

Schwartz A. 1977. Citrus silver mite Hernitarsonemus latus (Banks). Farming in South Africa. Department of Agricultural Technology Services, Republic of South Africa, Citrus Series, H. 14, 6 pp.

Schoonhoven L. M., Jermy T. \& van Loon J. J. A. 1998. Insect-Plant Biology. From physiology to Evolution. Chapman and Hall, London Skoracka A, Kuczynski L (2006)

Sikirou R., Afouda L., Zannou A., Komlan-Assogba F. \& Gbèhounou G. 2001. Diagnostic des problèmes phytosanitaires des cultures maraîchères au Sud-Bénin : Cas de la tomate, du piment, de l'oignon et du gombo, pp. 102-105. In: Acte de l'atelier scientifique Sud et Centre tenu du 12 au 13/12/2001 à Niaouli (Edité par B. P. Agbo, T. I. Isidore, A. Adjanohoun, I. Sagbohan, I. Gangbo, C. Bankolé, K. Igué et A. Mathers). INRAB, Cotonou, Bénin.

Tossounon G.A.R. \& Onzo A. 2015. Inventaire des acariens et insectes ravageurs associés à la culture du piment vert Capsicum chinense Jacq. (Solanales : Solanaceae) dans les communes de Kandi et Malanville au NordBénin. Annales de l'Université de Parakou, Série Sciences Naturelles et Agronomie, 5 : 1 11.

Waterhouse D. F. \& Norris K. R. (1987). Biological Control Pacific Prospects. Inkata Press, Melbourne, 454p.

Wilson L. 1997. Managing mites on cotton. http://www.cotton.pi.csiro.au/insects.htm? 2p.

Wilson L. \& Sadras V. 1997. Mite ecology on cotton. CRC Newsletter for the Research Extension Education Program 3 , http://www.cotton.pi.csiro.au/insects.htm?, 6p.

Zannou I.D. \& Hanna R., 2011. Clarifying the identity of Amblyseius swirskii and Amblyseius rykei (Acari: Phytoseiidae): are they two distinct species or two populations of one species? Experimental and Applied Acarology. 53 : 339-347. 